\title{
EFFECTS OF ALLOPURINOL ON GOUTY AND NON-GOUTY URIC ACID NEPHROPATHY
}

\author{
BY
M. A. OGRYZLO, M. B. UROWITZ, ${ }^{*}$ H. M. WEBER, ${ }^{*}$ and J. B. HOUPT
From the University of Toronto Rheumatic Disease Unit (Wellesley Hospital), and the Clinical Investigation Unit, Sunnybrook Hospital, Toronto, Canada

The kidneys are probably the only vital organs that are commonly affected by the precipitation of uric acid in patients with gout. Microscopic crystals of uric acid can sometimes be demonstrated in the collecting tubules, along with macroszopic concretions or calculi in the medulla and pelvis (Lichtenstein, Scott, and Levin, 1956; Talbott and Terplan, 1960; Gonick, Rubini, Gleason, and Sommers, 1965). Deposits of urate in the renal parenchyma may lead to a granulomatous reaction with foreign body giant cells and accumulations of chronic inflammatory cells, comparable to tophaceous lesions in other sites and obliterating the normal architecture. These are the more specific lesions of gouty nephropathy. Less specific, but perhaps more common, are the secondary changes affecting the renal parenchyma, generally regarded as indistinguishable from chronic nephritis, nephrosslerosis, or pyelonephritis. Progressive impairment of renal function with proteinuria and hypertension may reach an incidence of $\mathbf{2 5}$ per cent. or more. However, it is the vascular complications of atherosclerosis rather than renal insufficiency that account for the greatest mortality in gout.

More dramatic than these chronic renal changes is the acute uric acid nephropathy which frequently complicates myeloproliferative disorders during oncolytic therapy (Kritzler, 1958; Greenbaum and Stone, 1959; Frei, Bentzel, Reiseltach, and Block, 1963). Such cases are usually accompanied by an acute hyperuricaemia, resulting in the flooding of the urinary tract with excessive amounts of uric acid and giving rise to varying degrees of renal tubular obstruction or even complete urinary suppression, sometimes requiring haemodialysis (Reiselbach, Bentzel, Cotlove, Frei, and Freireich, 1964).

The present report concerns observations on

*Research Fellow of the Canadian Arthritis and Rheumatism Society. patients with varying types of renal dysfunction complicating gout or acute hyperuricaemia. The cases are drawn from a total of eighty subjects treated with allopurinol for periods up to 30 months.

\section{Materials and Methods}

Patients included in the study were initially admitted to the Clinical Investigation Unit, Sunnybrook Hospital, or to the Medical Service, Toronto General Hospital, witto subsequent follow-up as out-patients. Diets were res tricted only in foods with high purine content. Colchi cine was prescribed in maintenance doses $(0.6$ to $1.2 \mathrm{mg}$ daily) to patients having a history of frequently recurring attacks of acute gouty arthritis. Clearance data were $\mathrm{e}^{+}$ calculated on the basis of 24-hour collections of urine which tend to be somewhat lower than 2-hour data with enforced diuresis. Except where otherwise stated, the daily serum uric acid (SUA) and urinary uric acid (UUA) were determined colorimetrically by a modification of the method of Kern and Stransky (1937). Serum and urinaly creatinine were determined by the method of Hare as modified by Haugen and Blegen (1953). The oxypurines (hypoxanthine and xanthine) in the urine were measured by ultraviolet spectrophotometry using the enzymes uricase and xanthine oxidase (Kalckar, 1947) and calculating the difference in terms of uric acid. Haematolcgic and other laboratory procedures were carried out by standard procedures at appropriate intervals.

\section{Results}

The data to be presented will te considered in relation to the various aspects of renal dysfunction occurring in patients with gout and acute hyperuricaemia, together with observations on the effect of reducing the overall production of uric acid on these functions by means of the xanthine oxidase inhibitor allopurinol. The doses employed varied from 200 to $600 \mathrm{mg}$. daily (average 300 to $400 \mathrm{mg}$.). Larger doses were rarely required. 


\section{Clearance of Uric Acid}

In recent years there has been a revival of controversy concerning the apparent reduction in the clearance of uric acid $\left(\mathrm{C}_{\mathrm{urate}}\right)$ in patients with gout and the $\mathrm{C}_{\mathrm{urate}}$ /glomerular filtration rate (GFR) ratio, in relation to the serum concentration of uric acid (Wyngaarden, 1960; Ogryzlo, 1960; Sørensen, 1962; Bishop, 1962; Seegmiller, Grayzel, Howell, and Plato, 1962; Nugent, 1965; Gutman, 1965). Despite the higher levels of filtered urate load, the gouty kidney apparently excretes a smaller proportion of uric acid than does the non-gouty normal kidney. Thus, in 39 gouty subjects with a normal blood urea nitrogen (BUN) and a creatinine clearance (GFR) in excess of $75 \mathrm{ml} . / \mathrm{min}$., the mean clearance of uric acid was $4.8 \mathrm{ml} . / \mathrm{min}$. as contrasted to $7 \cdot 1 \mathrm{ml} . / \mathrm{min}$. in 23 normal subjects (Fig. 1). Similarly the $C_{\text {urate }}$ GFR ratio $\times 100$, was 4.4 in the gouty subjects as contrasted to 6.0 in the control group. Although the differences may appear small, they are nevertheless statistically significant. The effect of reducing the SUA concentration and therefore the filtered urate load to normal levels in gouty subjects by means of allopurinol is of considerable interest. The reduction in the SUA level by this means is accomplished solely by a reduction in the overall formation of uric acid and is accompanied by a decrease in the amount of uric acid excreted. No improvement in renal function has been observed, and the clearance of uric acid and $\mathrm{C}_{\text {urate }} / \mathrm{GFR}$ ratio remain relatively unchanged (Ogryzlo, 1965; Houpt, $\frac{D}{\omega}$. 1965). In a group of 75 subjects with gout and hyperuricaemia the $C_{\text {urate }}$ before treatment was $4 \cdot 7 \stackrel{\bar{ज}}{\stackrel{5}{5}}$ $\mathrm{ml} . / \mathrm{min}$. as compared with $4.8 \mathrm{ml} / \mathrm{min}$. while receiving allopurinol (Fig. 2). As the SUA was

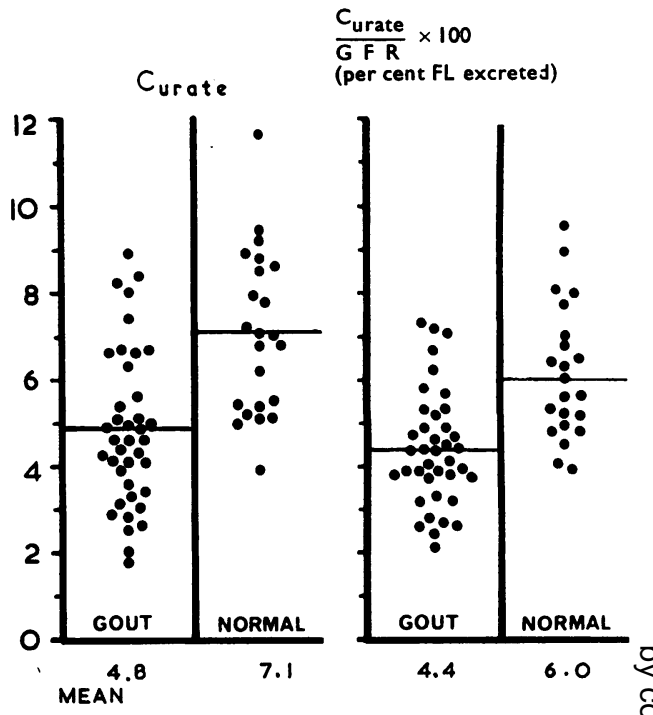

Fig. 1.-Comparison of the urate clearance and the $\mathrm{C}_{\text {urate }} / \mathrm{GFR}$ rato in 39 gouty subjects with normal renal function and 23 normal subjecs\$s.

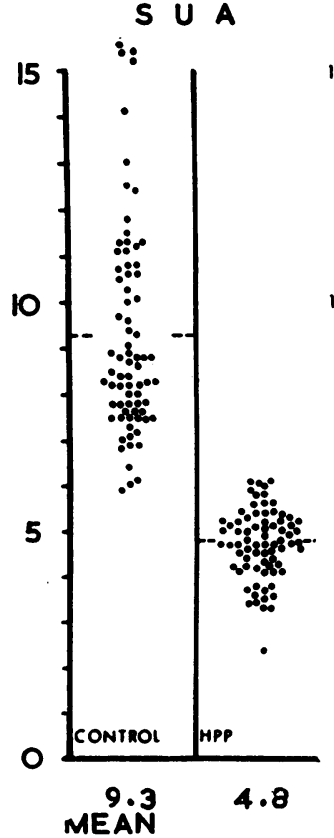

U U A

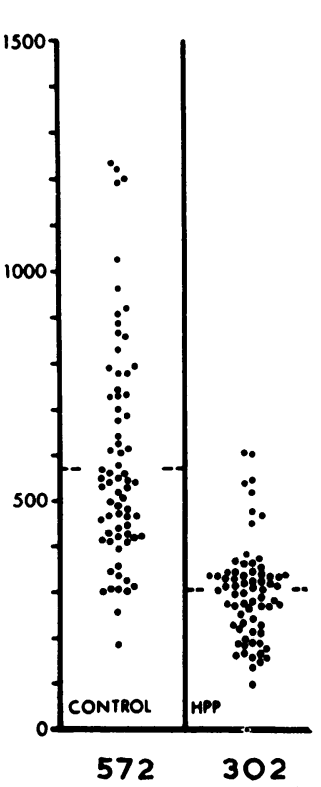

Curate

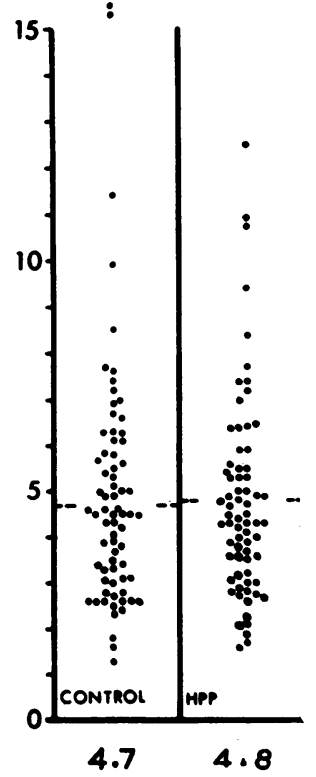

Fig. 2.- Response to allopurinol therapy in 75 patients with gout and hyperuricaemia, shown by fall in SUA level, UUA excretion. There was no change in the mean urate clearance when the gouty patients were rendered normouricaemic during treatment. 
reduced to normal levels in this group, it was apparent that the mean urinary uric acid (UUA) fell to subnormal levels, substantially below the level of excretion of normal subjects. This data therefore supports the concept that the gouty kidney excretes a smaller portion of its filtered urate load than does the normal kidney. While allopurinol will reduce the SUA to homeostatic levels, this reduction is accompanied by a subnormal UUA. To achieve homeostatic levels of both the SUA and UUA excretion, therefore, requires the enhancement of UUA excretion by some form of combined therapy with a uricosuric agent. Such combined treatment has obvious advantages in the management of patients with extensive tophaceous deposits, although it is not important in the majority of patients.

\section{Azotaemia}

Although the SUA level was reduced to normal with allopurinol (below $6.5 \mathrm{mg} . / 100 \mathrm{ml}$.) in an initial group of 75 consecutive subjects with gout and hyperuricaemia (Ogryzlo, Urowitz, Weber, and Houpt, 1966) as shown in Fig. 2, this observation would now appear not always to hold true. Patients with severe renal impairment and pronounced nitrogen retention may be exceptions.

In the past, the administration of uricosuric agents has not been practical in patients with moderate or severe azotaemia. UUA excretion would often remain unaffected or the treatment might even be accompanied by further impairment of renal function. In such situations allopurinol has obvious advantages and in most instances the SUA level can readily be reduced to normal. In fourteen consecutive patients with gout and azotaemia, normal SUA levels were achieved within a few days (Fig. 3, overleaf).

As mentioned before, there was no change in the clearance of urate, BUN, or other aspects of renal function. We have, however, encountered one exception in a 65-year-old male patient with gout and progressive renal impairment. $\mathrm{C}_{\mathrm{urate}}$ averaged $1.3 \mathrm{ml} . / \mathrm{min}$. and the $\mathrm{C}_{\text {creatinine }} 3.4 \mathrm{ml}$./min. (Fig. 4, overleaf).

Although the SUA level was reduced significantly below the control mean value of $14 \mathrm{mg} . / 100 \mathrm{ml}$., it was not possible to reduce the level below $7.5 \mathrm{mg}$./ $100 \mathrm{ml}$. with the usual dose of allopurinol.

On the basis of such observations and since the urate clearance remains substantially unaffected by allopurinol at varying levels of filtered urate load, it is possible to draw up a table which would enable one to predict the effect of such treatment, as well as the approximate level to which the SUA concentration may be expected to fall (Table). There would appear to be an obligatory minimum level, below which one cannot readily reduce the UUA excretion with the doses commonly in use. If one is to achieve homeostatic levels of SUA, therefore, the kidney must be capable of excreting this minimure amount at normal levels of SUA, based on it clearance capability. The average minimum UUA excretion on allopurinol therapy achieved by several groups (Rundles, Wyngaarden, Hitchings, Elion, and Silberman, 1963; Yü and Gutman, 1964; Ogryzlo and others, 1966) has varied from about

TABLE

URATE CLEARANCE VALUES ARE SHOWN IN RELATION TO VARYING LEVELS OF SERUM URIC ACID AND 24-HOUR URINARY URIC ACID EXCRETION. PATIENTS WITH URATE CLEARANCE VALUES ABOVE THE DIAGONAL SOLID LINE ARE NOT LIKELY TO ACHIEVE NORMAL SERUM URIC ACID LEVELS WITH STANDARD DOSES OF ALLOPURINOL.

\begin{tabular}{|c|c|c|c|c|c|c|c|c|c|c|c|c|c|c|c|c|c|}
\hline \multirow{2}{*}{$\begin{array}{l}\text { Urinary } \\
\text { Uric Acid } \\
\text { Excretion } \\
\text { (mg./day) }\end{array}$} & \multicolumn{17}{|c|}{ Serum Uric Acid Level (mg./100 ml.) } \\
\hline & 4 & 5 & 6 & 7 & 8 & 9 & 10 & 11 & 12 & 13 & 14 & 15 & 16 & 17 & 18 & 19 & 20 \\
\hline 50 & 0.87 & 0.69 & 0.58 & $0 \cdot 50$ & 0.43 & $0 \cdot 39$ & $0 \cdot 35$ & $0 \cdot 32$ & 0.29 & $0 \cdot 27$ & 0.25 & 0.23 & 0.22 & $0 \cdot 20$ & 0.19 & $0 \cdot 18$ & $0 \cdot 17$ \\
\hline 100 & $1 \cdot 74$ & $1 \cdot 39$ & $1 \cdot 16$ & 0.99 & 0.87 & 0.77 & 0.69 & $9 \cdot 63$ & $0 \cdot 58$ & 0.53 & 0.50 & 0.46 & 0.43 & 0.41 & $0 \cdot 39$ & $0 \cdot 37$ & 0.35 \\
\hline 150 & $2 \cdot 60$ & $2 \cdot 08$ & $1 \cdot 74$ & 1.49 & $1 \cdot 30$ & $1 \cdot 16$ & $1 \cdot 04$ & 0.95 & 0.87 & 0.80 & 0.74 & 0.69 & 0.65 & 0.61 & 0.58 & 0.55 & 0.52 \\
\hline 200 & $-3 \cdot 47$ & $\mathbf{2 \cdot 7 8}$ & $2 \cdot 31$ & $1 \cdot 98$ & $1 \cdot 74$ & $1 \cdot 54$ & $1 \cdot 39$ & $1 \cdot 26$ & $1 \cdot 16$ & $1 \cdot 07$ & 0.99 & 0.93 & $0 \cdot 87$ & 0.82 & $0 \cdot 77$ & $0 \cdot 73$ & 0.69 \\
\hline 250 & $4 \cdot \overline{34}$ & $3 \cdot 47$ & $2 \cdot 89$ & $2 \cdot 48$ & $2 \cdot 17$ & 1.93 & $1 \cdot 74$ & $1 \cdot 58$ & $1 \cdot 45$ & $1 \cdot 34$ & $1 \cdot 24$ & $1 \cdot 16$ & $1 \cdot 08$ & $1 \cdot 02$ & 0.96 & 0.91 & 0.87 \\
\hline 300 & $5 \cdot 211$ & $\underline{4} \cdot \underline{17}$ & $3 \cdot 47$ & $2 \cdot 98$ & $2 \cdot 60$ & $2 \cdot 31$ & $2 \cdot 08$ & $1 \cdot 89$ & $1 \cdot 74$ & $1 \cdot 60$ & $1 \cdot 49$ & $1 \cdot 39$ & $1 \cdot 30$ & $1 \cdot 22$ & $1 \cdot 16$ & $1 \cdot 10$ & $1 \cdot 04$ \\
\hline 350 & 6.08 & $\overline{4} \cdot \overline{86}$ & 4.05 & $3 \cdot 47$ & $3 \cdot 04$ & $2 \cdot 70$ & $2 \cdot 43$ & $2 \cdot 21$ & $2 \cdot 03$ & $1 \cdot 87$ & $1 \cdot 74$ & $1 \cdot 62$ & $1 \cdot 52$ & $1 \cdot 43$ & $1 \cdot 35$ & $1 \cdot 28$ & $1 \cdot 22$ \\
\hline 400 & $6 \cdot 94$ & $5 \cdot 56$ & $4 \cdot 63 i$ & $3 \cdot 97$. & $3 \cdot 47$ & $3 \cdot 09$ & $2 \cdot 78$ & $2 \cdot 52$ & $2 \cdot 31$ & $2 \cdot 14$ & $1 \cdot 98$ & $1 \cdot 85$ & $1 \cdot 74$ & $1 \cdot 63$ & $1 \cdot 54$ & $1 \cdot 46$ & $1 \cdot 39$ \\
\hline 450 & $7 \cdot 81$ & $6 \cdot 25$ & $5 \cdot 21$ & $4 \cdot 46 !$ & 3.91 & $3 \cdot 47$ & $3 \cdot 12$ & $2 \cdot 84$ & $2 \cdot 60$ & $2 \cdot 40$ & $2 \cdot 23$ & $2 \cdot 08$ & 1.95 & $1 \cdot 84$ & $1 \cdot 74$ & $1 \cdot 64$ & $1 \cdot 56$ \\
\hline 500 & $8 \cdot 68$ & $6 \cdot 94$ & $5 \cdot 79$ & $4 \cdot 96$ & $\overline{4} \cdot \overline{34} \mathrm{i}$ & $3 \cdot 86$ & $3 \cdot 47$ & $3 \cdot 16$ & $2 \cdot 89$ & $2 \cdot 67$ & $2 \cdot 48$ & $2 \cdot 31$ & $2 \cdot 17$ & $2 \cdot 04$ & 1.93 & $1 \cdot 83$ & 1.74 \\
\hline 550 & $9 \cdot 55$ & $7 \cdot 64$ & $6 \cdot 37$ & $5 \cdot 46$ & $4 \cdot 77$ & $\overline{4} \cdot \overline{24}$ & 3.82 & $3 \cdot 47$ & $3 \cdot 18$ & $2 \cdot 94$ & $2 \cdot 73$ & $2 \cdot 55$ & $2 \cdot 39$ & $2 \cdot 25$ & $2 \cdot 12$ & $2 \cdot 01$ & 1.91 \\
\hline 600 & 10.40 & $8 \cdot 33$ & $6 \cdot 94$ & 5.95 & $5 \cdot 21$ & $4 \cdot 63$ & L4.17 & $3 \cdot 79$ & $3 \cdot 47$ & $3 \cdot 20$ & $2 \cdot 98$ & $2 \cdot 78$ & $2 \cdot 60$ & $2 \cdot 45$ & $2 \cdot 31$ & $2 \cdot 19$ & $2 \cdot 08$ \\
\hline 650 & $11 \cdot 30$ & 9.03 & $7 \cdot 52$ & 6.45 & $5 \cdot 64$ & $5 \cdot 01$ & $4 \cdot 51$ & $4 \cdot 10$ & $3 \cdot 76$ & $3 \cdot 47$ & $3 \cdot 22$ & $3 \cdot 01$ & $2 \cdot 82$ & $2 \cdot 65$ & $2 \cdot 51$ & $2 \cdot 37$ & $2 \cdot 26$ \\
\hline 700 & $12 \cdot 20$ & $9 \cdot 72$ & $8 \cdot 10$ & $6 \cdot 94$ & $6 \cdot 08$ & $5 \cdot 40$ & $4 \cdot 86$ & $4 \cdot 4 \overline{2}$ & $4 \cdot 05$ & $3 \cdot 74$ & $3 \cdot 47$ & $3 \cdot 24$ & 3.04 & $2 \cdot 86$ & $2 \cdot 70$ & $2 \cdot 56$ & $2 \cdot 43$ \\
\hline
\end{tabular}




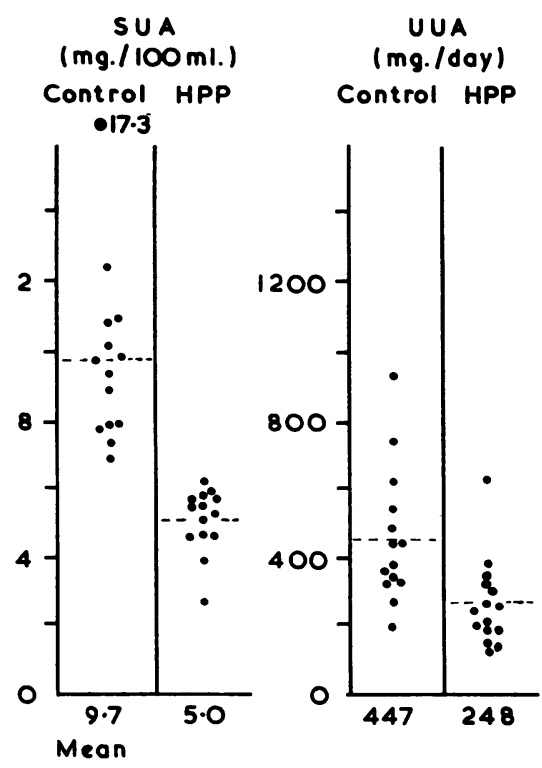

\author{
Curate \\ (ml./min.) \\ BUN \\ Control HPP \\ ( $\mathrm{mg} . / 100 \mathrm{ml}$. \\ Control HPP
}
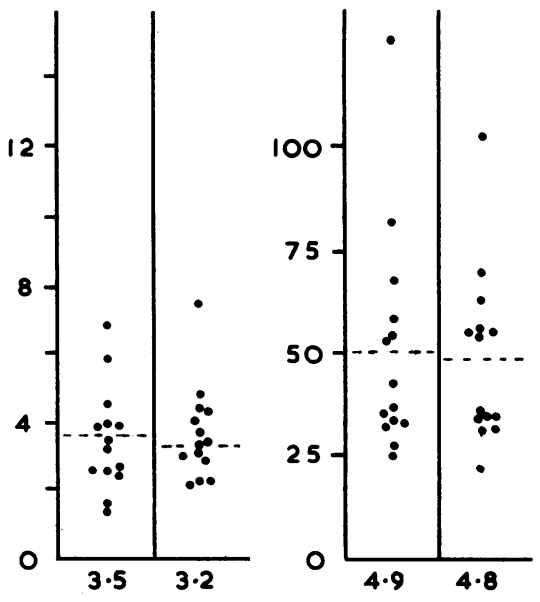

Fig. 3.-Response to allopurinot therapy in fourteen gouty subes

jects with azotacmia, shown b

fall in SUA levia, shown

fall in SUA level and UUA

excretion. There was no signifio

cant chanze in the mean values

for urate clearance or BUN during treatment.
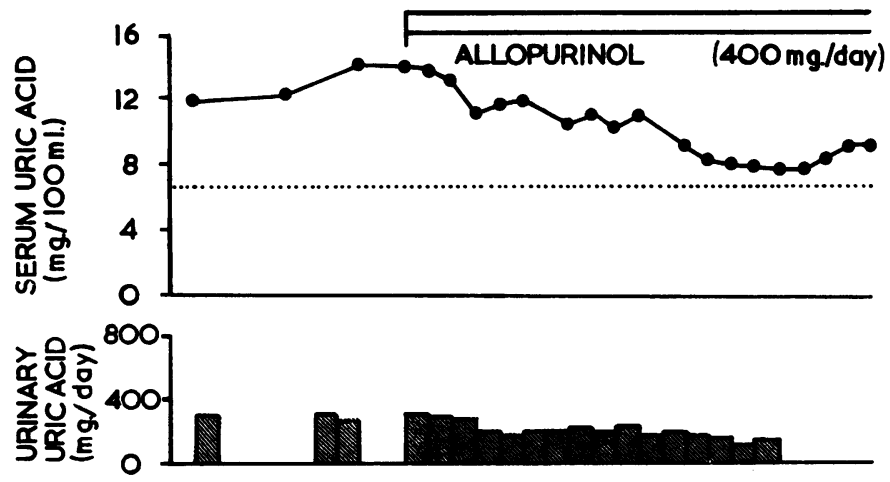

Fig. 4.-Response to allopurino in a gouty subject with renal failure (not included in Fig. 2) The SUA dropped from 14 to $7.5 \mathrm{mg} . / 100 \mathrm{ml}$. but did not reach normal levels. U U A decreased from 290 to 150 mg./day but the urate clearance remainea unchanged.

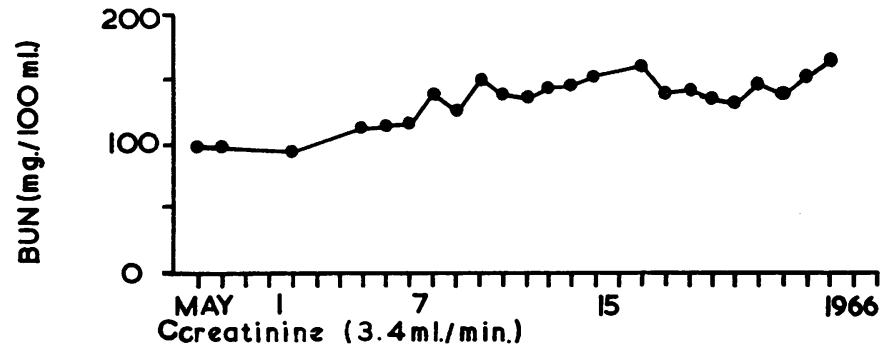


150 to $300 \mathrm{mg}$. (mean 200)/day (over-excretors excluded). The critical level of $\mathrm{C}_{\text {urate }}$ with which normal concentrations of SUA can be maintained at this excretion level (within certain variations) is about $1.8 \mathrm{ml}$./min. Thus, with $\mathrm{C}_{\text {urate }}$ values of $2 \mathrm{ml}$./min. or higher, it may be predicted that normal SUA levels can readily be achieved. However, normal serum concentrations cannot readily be achieved with clearance values below $1.8 \mathrm{ml}$./ min. For example, a patient with a SUA concentration of $16 \mathrm{mg}$. $/ 100 \mathrm{ml}$. and a UUA excretion of $300 \mathrm{mg}$./day before allopurinol therapy can be expected to excrete a lesser amount when allopurinol therapy is instituted. With a $\mathrm{C}_{\text {urate }}$ of $1.3 \mathrm{ml} . / \mathrm{min}$., it can be predicted that the UUA excretion may drop to an obligatory minimum of approximately $150 \mathrm{mg}$./day, while the SUA concentration may be expected to fall to approximately $8 \mathrm{mg} . / 100 \mathrm{ml}$. Lower SUA levels are not likely to be attained, and may even remain substantially higher. However, since it is very unusual to encounter $C_{u r a t e}$ values below $2 \mathrm{ml}$./min., except in situations of endstage renal insufficiency, normal SUA levels can be obtained in virtually all patients receiving allopurinol.

\section{Acute Uric Acid Nephropathy}

A very important contribution of allopurinol therapy has been in the management and prevention of acute uric acid nephropathy, apt to occur during the chemotherapy of neoplastic haemopoietic diseases.

This is illustrated by the case of a 50 -year-old female with acute or chronic myeloid leukaemia, treated with 6-mercaptopurine and busulphan (Fig. 5, overleaf, p. 678). As the white cell count and platelets fell to low levels, the BUN (17 mg. $/ 100 \mathrm{ml}$. on admission) had risen to 97 mg. $/ 100 \mathrm{ml}$. At this point the SUA had risen to 15.9 $\mathrm{mg} . / 100 \mathrm{ml}$. and the UUA to $970 \mathrm{mg}$./day, and the urine volume had dropped to $300 \mathrm{ml}$./day. On allopurinol the SUA fell abruptly to $2 \mathrm{mg}$. $/ 100 \mathrm{ml}$. with a reduction in UUA to $250 \mathrm{mg}$./day. This was accompanied by a diuresis and a progressive decrease in the BUN to normal levels.

A similar dramatic response was achieved in two other patients.

Results of this type are very dramatic indeed. Presumably the suppression of uric acid formation reduces the load of uric acid presented to the renal tubules, and in conjunction with a liberal fluid intake and alkali permits the precipitated uric acid to go into solution again. Tubular obstruction may thereby be relieved. The success of this form of treatment obviously depends on the ultimate ability of the kidney to resume normal filtration of urine. However, the obvious question arises whether complications of this type may not be completely eliminated by instituting allopurinol before oncolytic therapy.

\section{Uric Acid Urolithiasis}

Precipitation of uric acid with the formation of uric acid calculi and subsequent renal colic, is a frequent complication in patients with primary and secondary gout, the incidence being estimated at 20 and 40 per cent. respectively (Gutman, 1963, 1964). Uricosuric therapy may further aggravate the situation unless adequate fluid and alkali are taken, since the UUA excretion may be substantially increased. Conversely, the suppression of uric acid formation by allopurinol may be expected to reduce the incidence of this complication.

Allopurinol was administered to 27 patients with a history of gout and uric acid stones (Fig. 6). During the 3-year period immediately preceding allopurinol therapy, this group of 27 patients had suffered 63 episodes of renal colic due to uric acid stones as well as numerous episodes of passing "gravel" in the urine. They had also been subjected to fourteen surgical procedures for removal of stones. In the course of 30 months of allopurinol therapy, without regard to fluid intake or alkalinization of the urines not a single instance of uric acid stone formation has
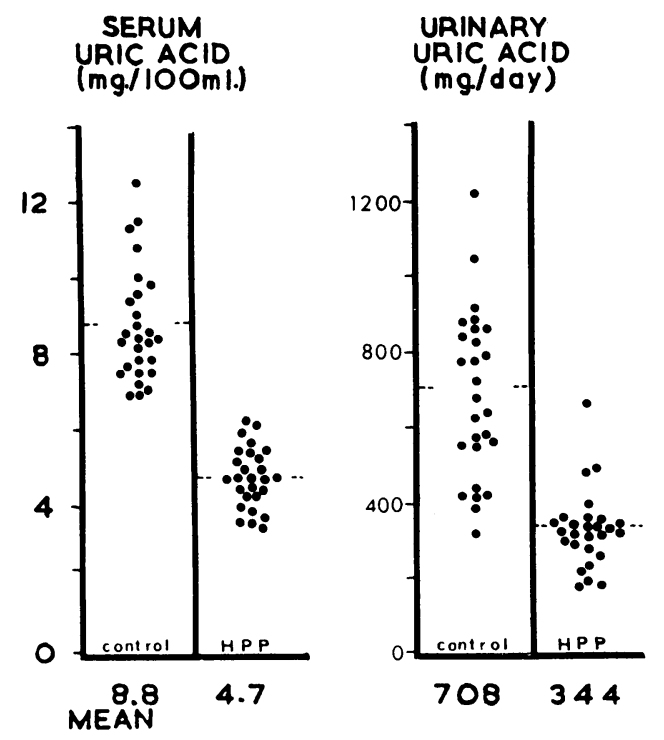

Fig. 6.--Response to allopurinol in 27 patients with gout and uric acid stones. No further stones were passed during allopurinol therapy. 

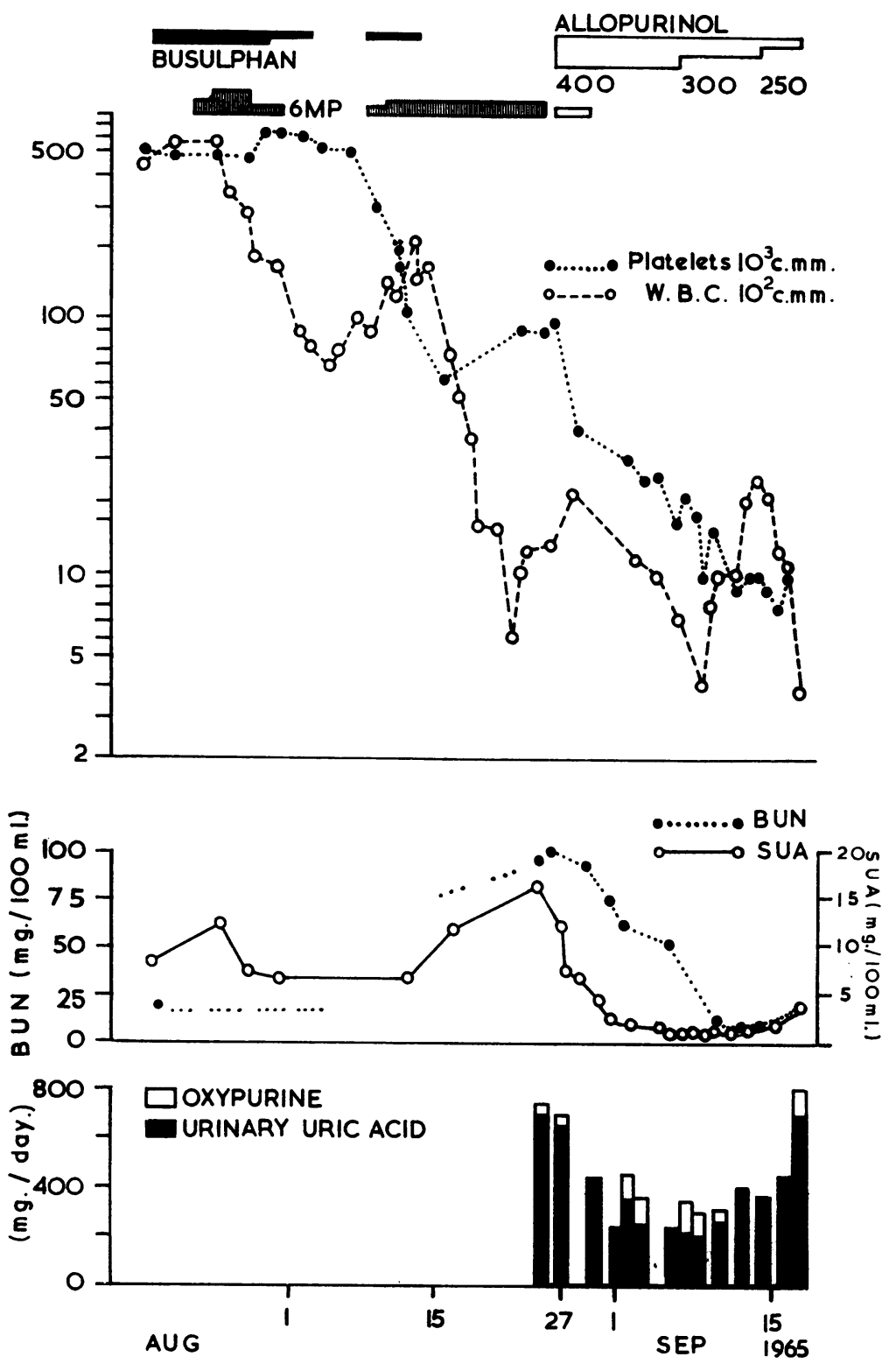

Fig. 5.-Acute uric acid nephropathy in a leukaemic patient during oncolytic therapy. Note the rise in the SUA and BUN, and their prompt return to normal values after allopurinol administration.

been encountered. One patient on combined allopurinol and sulphinpyrazone therapy suffered an episode of mild flank pain, but no stone was passed. The significance of these observations is of considerable importance, since it would now appear that the problem of uric acid urolithiasis might be virtually eliminated by this form of treatment.
An interesting observation has been made in on additional patient who repeatedly passed calciußf oxalate stones and "gravel". Because he had "a SUA of $7.8 \mathrm{mg} . / 100 \mathrm{ml}$. and excreted UUA at the rate of $641 \mathrm{mg}$./day, allopurinol $400 \mathrm{mg}$. daily wás prescribed. There was no clinical history of gou The SUA dropped to $3.6 \mathrm{mg} . / 100 \mathrm{ml}$. and the UUT 
to $322 \mathrm{mg}$./day. Surprisingly, this patient ceased to form further calcium oxalate stones or to pass "gravel" during 20 months of therapy. Conceivably, the reduction in UUA may decrease the risk of uric acid precipitates forming a nidus for subsequent calcium oxalate stone formation, or alternatively it may have a favourable effect on the solubility of calcium oxalate in the urine, since neither the excretion of calcium or oxalate are affected by allopurinol.

\section{Combined Medications}

Brief comment should be made concerning two aspects of renal function associated with the administration of other medications on the observed action of allopurinol. There would appear to be no incompatibility in the use of uricosuric agents along with allopurinol, and the net effect on the SUA level would appear to be additive.

Thus, in a 58-year-old male with tophaceous gout, the serum uric acid was substantially reduced with allopurinol alone, but its concentration was decreased even further when the uricosuric agent sulphinpyrazone was added (Fig. 7). This was shown during two periods of combined therapy. The amount of UUA excreted and $\mathrm{C}_{\text {urate }}$ were increased during combined therapy and accounted for the greater effectiveness of the combined form of treatment. However, the administration of a uricosuric agent during allopurinol therapy appeared to have the effect of inhibiting to a varying degree, the excretion of oxypurines in the urine, resulting in slightly reduced oxypurine excretion during the periods of combined therapy.

The effect of salicylate administration along with allopurinol is also of some interest, particularly in regard to possible misinterpretation of results, especially when the colorimetric procedures are employed for the measurement of uric acid (Fig. 8, overleaf).

There would appear to be no problem in regard to the reading of the SUA levels, which show good correlation between the colorimetric and the uricase procedures. In large doses, salicylates may further reduce the SUA concentration by virtue of their uricosuric action. However, in measuring the urinary excretion, while the two methods show good correlation when no salicylate is administered, there is a distinct spread between the values obtained by the two methods after salicylates have been added. With low doses of salicylate (40 gr. daily), this discrepancy may be as much as $100 \mathrm{mg}$./day, while with larger doses ( $80 \mathrm{gr}$. daily) it may be as much as 400 to $500 \mathrm{mg}$./day. The falsely high values in the urine
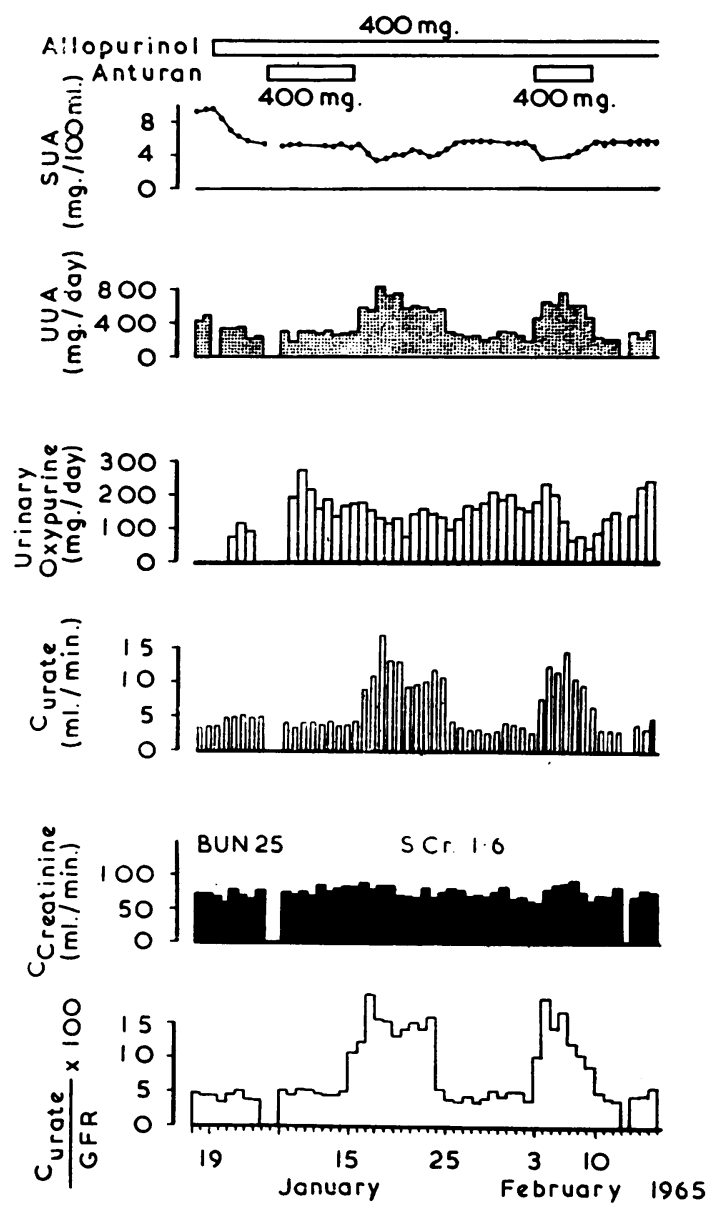

Fig. 7.-Effect of uricosuric agents on oxypurine excretion. Note the reduction in oxypurine (xanthine and hypoxanthine) excretion during two periods of combined allopurinol-anturan therapy.

obtained with the colorimetric procedure during salicylate administration are probably due to the fact that the colorimetric procedure also measured other breakdown products of salicylates, presumably gentisic acid, which had not been removed.

\section{Summary}

In spite of the high level of the serum uric acid (SUA) and the greater filtered uric acid load presented to the tubules, gouty subjects as a group, have lower levels of urate clearance and $\mathrm{C}_{\text {urate }} / \mathrm{GFR}$ ratios than non-gouty control subjects. The data presented indicate that $C_{\text {urate }}$ and the $C_{\text {urate }} / G F R$ ratio remain substantially unchanged during therapy with allopurinol. When normal SUA levels were achieved, the gouty subjects persisted in having 


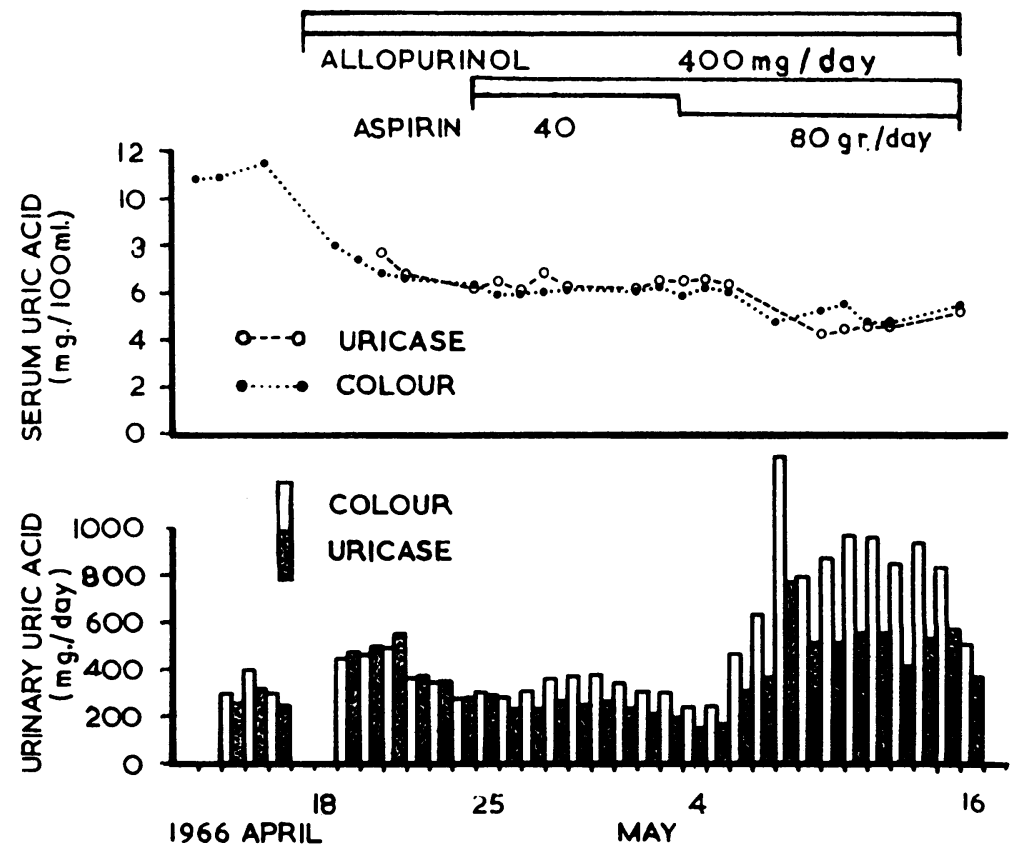

Fig. 8.-Effect of salicylates combined with allopurinol in a gouty subject. Note the good correlation of the SUA by the colorimetric and uricase methods, but the falsely high values in the UUA by the colorimetric method. A uricosuric effect is also shown with the larger doses of salicylate.

lower $\mathrm{C}_{\text {urate }}$ and $\mathrm{C}_{\text {urate }} / \mathrm{GFR}$ ratios, and excreted a smaller amount of UUA than non-gouty controls. The reduction in the SUA concentration, therefore, was accomplished solely by a reduction in the amount of uric acid formed and requiring to be excreted.

Allopurinol has been highly effective in reducing hyperuricaemia even in the presence of chronic renal insufficiency. It is particularly valuable in the treatment of acute uric acid nephropathy complicating oncolytic therapy in patients with myelopro- liferative diseases. Recurrent uric acid urolithia in 27 patients was virtually eliminated after the suppression of uric acid formation by allopurinol therapy. Reduction in uric acid excretion with allopurinol also resulted in a cessation of calcium oxalate stone formation in one patient.

The authors are indebted to Mrs. S. Crier and Missō V. Wyhovsky for the many determinations of uric acid 3 and creatinine, and to the many physicians who have referred patients for this study. 